Results Chemical exposures as indicated by median and geometric mean values were low (e.g. 2.5 and 4.4 ppb respectively for methyl bromide) and none exceeded the NZ WES, although formaldehyde exceeded the TLV in 48 (26.2\%) samples. The AMV-TLV threshold of 1 was exceeded in 54 (29.5\%) samples. Major differences between the occupational groups (container, log and retail workers and fumigators) were not found. Time spent inside the container was significantly and positively associated with ethylene oxide, C2-alkylbenzenes and acetaldehyde, but this was based on a small number of detectable observations (non-detects 52.0\%-95.9\%) and for one chemical (methyl bromide, $\mathrm{n}=94$ ) we found inverse associations.

Conclusion This study has shown that personal exposures measured over an 8 hour working day were low, with the exception of formaldehyde, and do not confirm earlier suggestions (based on measurements inside closed containers) that they regularly exceed exposure limits. Association with time spent unloading containers was weak suggesting that exposures may result from short peak exposures rather than continuous low level exposure.

\section{D.4 OCCUPATIONAL EXPOSURE TO FORMALDEHYDE IN FRANCE IN 2015}

'Laurène Delabre*, 'Loïc Garras, ${ }^{1}$ Marie Houot, ${ }^{1,2}$ Corinne Pilorget. ${ }^{1}$ Santé Publique France, Saint-maurice, France; ${ }^{2}$ University Claude Bernard Lyon1, Ifsttar, UMRESTTE, UMR T_9405, Lyon, France

\subsection{6/OEM-2019-EPI.26}

Context Formaldehyde has been used in a large range of activities for decades. It was classified as human carcinogenic in 2004 by the International Agency for Research on Cancer and in France, formaldehyde has been regulated as a carcinogen since 2007.

The aim of this work is to describe the occupational exposure to formaldehyde in France in 2015 and to identify the most exposed activities.

Method A formaldehyde job-exposure matrix, developed under the MATGÉNÉ programme, provides an exhaustive and retrospective exposure assessment for all jobs in France. These data were linked with the 2015 French census, annually elaborated from 2013 to 2017, to estimate the occupational exposure prevalence by gender, occupation, industries and worker status. Results In 2015, 90000 workers were occupationally exposed to formaldehyde (3.5\% of the French population at work), 60600 (4.6\%) among men and 29400 (2.4\%o) among women.

The industries with the highest prevalence exposure rate were for men veterinary area $(8.8 \%)$, wood industries $(7.7 \%)$ and agriculture (4.8\%); for women, veterinary area $(7.3 \%)$, fishing and aquaculture (5.3\%) and agriculture (5.1\%).

The exposed workers are retrieved in agriculture (26\%), healthcare sectors $(13,5 \%)$ and specialized construction work (12\%) for employees and agriculture (69.6\%), specialized construction work $(13.5 \%)$ and other personal services $(8.1 \%)$ for people with worker status different (mostly self-employed).

Conclusion These results, the first describing the occupational exposure to formaldehyde in France, according to gender for the entire working population, show the importance of regulation in the variation of occupational exposure prevalence rates. This information will help in the surveillance of this occupational risk and to prioritize prevention actions.
01D.5

NON-DETECTS IN OSHA'S IMIS DATABANK: ARE THEY SHORT-TERM OR SHIFT-LONG SAMPLES?

${ }^{1}$ Philippe Sarazin*, ${ }^{2}$ George Luta, ${ }^{3}$ Igor Burstyn, ${ }^{4}$ Laurel Kincl, ${ }^{5}$ Jérôme Lavoué. ${ }^{1}$ Institut de recherche Robert-Sauvé en santé et en sécurité du travail (IRSST), Montréal, Canada; ${ }^{2}$ Georgetown University, Washington, USA; ${ }^{3}$ Drexel University, Philadelphia, USA; ${ }^{4}$ Oregon State University, Corvallis, USA; ${ }^{5}$ Université de Montréal, Montréal, Canada

\subsection{6/OEM-2019-EPI.27}

Objectives The Integrated Management Information System (IMIS) is the largest multi-industry source of exposure measurements available in North America. However, the lack of information on the censoring value (that depends on duration of sampling) of non-detected (ND) measurements considerably limits the usefulness of this databank. Released in 2010, the Chemical Exposure Health Database (CEHD) contains analytical results and measurement details, including duration of sampling for some of the records in IMIS. We assessed which ND results stored in IMIS are short-term (ST), and which are shift-long (LT) samples, based on information available in CEHD.

Methods We analyzed exposure measurements for 54 agents from 1984-2009 $(n=238,826)$. First, we calculated kappa coefficients $(\mathrm{\kappa})$ for each agent to investigate the agreement between the exposure type of IMIS detected records (already indicated as ST or LT, i.e. selected by OSHA officers) and the exposure type suggested by sampling duration found in CEHD. If $\mathrm{k}$ exceeded 0.3 for an agent, we employed classification and regression trees (CART) models to predict whether the ND results from IMIS should be classified as ST or LT samples. CART was developed using CEHD and applied to IMIS, relying on predictors common to both databanks: industry, reason for inspection, scope of inspection, region, union status, and year of sampling.

Results The median proportion of ND results per agent was $37 \%$ (interquartile range $(\mathrm{IQR})=22 \%-62 \%)$. The median $\mathrm{K}$ was $0.45 \quad(\mathrm{IQR}=0.37-0.64)$ and $0.03 \quad(\mathrm{IQR}=0.01-0.16)$ for solvents/gases and metals/isocyanates, respectively. Solvents $(n=22)$ and gases $(n=7)$ were selected for CART modeling. Industry was the most important predictor variable in classifying ND results into either ST or LT.

Conclusions This novel approach can be used to assign a censoring value to ND results, thus allowing more accurate inference about distribution of exposure levels in IMIS.

\section{Work Organisation}

\section{E.2 INFLUENCE OF WORK ORGANIZATION AND ENVIRONMENT ON HEALTH AND PRODUCTIVITY OUTCOMES AMONG CONSTRUCTION APPRENTICES: A TOTAL WORKER HEALTH ${ }^{\circledR}$ APPROACH}

Ann Dale*, 1,2 Bradley Evanoff, 2,3 Diane Rohlman, 1,2 Jaime Strickland, ${ }^{2,3}$ Kevin Kelly. ${ }^{1}$ Washington University School of Medicine - General Medical Sciences, St. Louis, USA; ${ }^{2}$ Healthier Workforce Center of the Midwest, lowa City, USA; ${ }^{3}$ University of lowa College of Public Health, lowa City, USA

\subsection{6/OEM-2019-EPI.28}

Introduction Construction is a hazardous industry, with wellrecognized risks of traumatic injury and high physical demands. Other threats to construction workers' health and well-being occur from work organization and work environment factors, including precarious employment, long 
commutes, long work hours, and employer policies regarding health and safety. These non-traditional hazards have been associated with injury and illness, psychosocial stress, and unhealthy behaviors including poor diet and smoking. The cumulative impacts of both traditional and non-traditional hazards on the health and well-being of construction workers are largely unknown.

Methods We conducted annual surveys among apprentice construction workers to identify relationships between four study domains: work organization and environment, health behaviors, health outcomes, and work outcomes.

Results 963 baseline surveys were completed and returned by apprentice construction workers $(90 \%$ response rate, mean age 28). Preliminary analyses examined associations between work organization factors and four self-reported outcomes: lower work ability, lower productivity, higher rates of missed days of work due to injury, and use of prescription pain medication. We found that all four outcomes were associated with high job demands, low supervisor support, and low job security. Other factors associated with one or more outcomes included low job security, mandatory overtime, low coworker support, and low foreman supervision of safety. Compared to commercial construction workers, those in residential construction reported higher use of pain medication and higher rates of missed days due to work injuries. One year follow-up data from 901 workers are now being analyzed; we will present results of relationships between the four study domains.

Discussion Cross-sectional data highlight non-traditional worksite health risks, and suggest potential interventions to improve heath behaviors and outcomes among construction workers.

\section{E.3 EVALUATION OF THE NORWEGIAN AGREEMENT ON A MORE INCLUSIVE WORKING LIFE: SICKNESS ABSENCE IN INDIVIDUALS WITH MUSCULOSKELETAL AND PSYCHOLOGICAL DIAGNOSES}

Rachel L Hasting*, Therese N Hanvold, Suzanne L Merkus, Petter Kristensen, Ingrid S Mehlum. National Institute of Occupational Health, Oslo, Norway

\subsection{6/OEM-2019-EPI.29}

Objectives The Norwegian Agreement on a More Inclusive Working Life (the IW Agreement) was introduced in 2001 amid efforts to reduce sickness absence (SA) and increase work participation. Little research has been done on the effects of this agreement. The study's aim was to compare SA before and after introduction of the IW Agreement.

Methods Data from several national registries involving 115,119 individuals born in Norway 1967-1976 was used. Individuals were classified using ICPC-2 codes into musculoskeletal (code L) and mental health (code P) diagnosis groups. A difference-in-differences method using logistic regression was used to compare the difference in one year risk of SA lasting $>16$ days in individuals working in IW companies relative to non-IW companies in 2000 and 2005. Analyses were adjusted for age, gender, and industry. Standard errors were clustered at the individual level and average marginal effects were calculated with 95\% confidence intervals. Gender and industry specific models were also estimated.

Results There was no significant association between the IW Agreement and risk of SA in the overall musculoskeletal diagnosis group (-0.4 percentage points (PP), CI -0.9,0.1), but women had significantly lower risk of SA (-0.8 PP, CI $-1.5,-$
0.1). There was no association between the IW Agreement and risk of SA in the mental health diagnosis group (overall: $0.0 \mathrm{PP}, \mathrm{CI}-0.3,0.3)$. Significant associations were found in the electricity (males: 5.2 PP, CI 0.4,10.0) and wholesale/retail sectors (females: $-4.9 \mathrm{PP}, \mathrm{CI}-8.9,-1.0$ ) for musculoskeletal diagnoses, and in the financial/real estate sector (overall: 1.6 PP, CI 0.2,2.9, females: 3.1 PP, CI 0.2,5.9) for mental health diagnoses.

Conclusions Women in companies with an IW Agreement had a significant reduction in one year risk of SA for musculoskeletal diagnoses. Gender and industry may modify the association between the IW Agreement and SA in musculoskeletal and mental health diagnoses.

\section{$01 E .4$ ELECTRONIC WASTE RECYCLING IN QUÉBEC, CANADA: HIRING PRACTICES AND OCCUPATIONAL HEALTH AND SAFETY MANAGEMENT}

${ }^{1}$ Sylvie Gravel, ${ }^{2,3}$ Daniel Côté, ${ }^{4}$ Stéphanie Gladu, 2,5 France Labrèche*. ${ }^{1}$ School of Management Sciences, Université du Québec à Montréal (UQAM), Montréal, Canada; ${ }^{2}$ Institut de recherche Robert-Sauvé en santé et en sécurité du travail (IRSST), Montréal, Canada; ${ }^{3}$ Anthropology Department, Université de Montréal, Montréal, Canada; ${ }^{4}$ nstitute of Environmental Sciences, UQAM, Montréal, Canada; ${ }^{5}$ School of Public Health, Université de Montréal, Montréal, Canada

\subsection{6/OEM-2019-EPI.30}

Background and objective Electronic waste recycling (e-recycling) has received little attention from an occupational health and safety (OHS) perspective. Our objective was to describe hiring and OHS management practices in a sample of formal e-recycling facilities.

Methods Within a cross-sectional study of exposure of e-recycling workers to various contaminants, we conducted semistructured face-to-face interviews with a sample of 26 workers and 6 managers, employed in four companies. Thematic analyses, followed by a matrix analysis based on the companies' missions were conducted on the recorded interviews.

Results Three companies are small enterprises: one receives young offenders/ex-prisoners for up to six months of vocational internships; another is a private company recruiting its workforce through governmental programs integrating people with chronic health problems; the third, a family business, mainly employs workers within neighbouring communities. Lastly, a medium-sized unionized company recruits its employees through staffing agencies, offering permanent jobs to the best candidates after a three-month trial period. Most participants were male, aged between 20-50 years old, and had not completed high school, except for a few recent immigrants with graduate degrees. Regarding occupational hazards in their workplace, $40 \%$ of interviewees reported chemicals, $31 \%$ mentioned the danger of being struck by lift trucks, and less than $25 \%$ identified toxic vapours, inappropriate protective personal equipments (PPEs), cuts, dusts, musculoskeletal or back pain. Some workers expressed concern about the pace of work (and resulting stress), which they identified as an injury risk factor. None of the participants received any mentoring upon entering the job. Agency workers had inferior wages and did not have access to the same OHS preventive practices or PPEs as regular workers.

Conclusions In our sample, OHS management practices varied according to the employment relationship, although workers are exposed to similar working conditions. Working conditions in the growing e-recycling industry need our attention. 